\title{
Delamination of impacted composite structures by cohesive zone interface elements and tiebreak contact
}

Research Article

\author{
Fatih Dogan ${ }^{1}$, Homayoun Hadavinia ${ }^{1 *}$, Todor Donchev ${ }^{1}$, Prasannakumar S. Bhonge ${ }^{2}$ \\ 1 Material Research Centre, SEC Faculty, Kingston University, London, SW15 3DW, UK \\ 2 Department of Mechanical Engineering, Wichita State University, Wichita, KS 67260, USA
}

Received 28 April 2012; accepted 21 May 2012

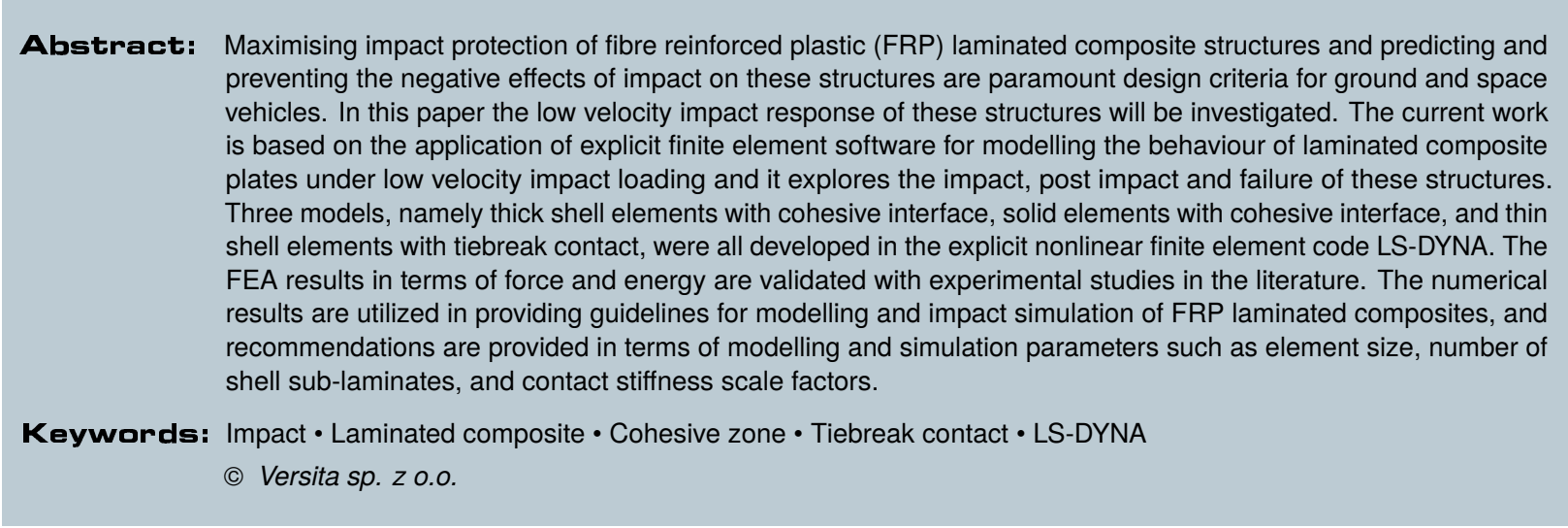

\section{Introduction}

Fibre reinforce polymer (FRP) composite materials are in common use in many ground and space vehicles particularly in aircraft, sailplanes, marine structures such as submarines, racing yachts and also in sport equipments such as golf club and bicycle since they allow a lighter structure, which increases efficiency by reducing weight, fuel consumption and weight-based maintenance. FRP composite materials also have a better fatigue performance relative to metals and furthermore their resistance to corro-

*E-mail: h.hadavinia@kingston.ac.uk sion is superior to metals. These factors reduce scheduled maintenance and increase productive time.

The impact resistance of FRP composites is good and they can be designed for easy visual inspection. They can be repaired easily, e.g. minor damage in aeroplane structures can be repaired at the gate in a very short time. Larger damaged structures can be repaired similarly to today's aircraft by using bolted repairs or a bonded repair. Amongst many fibre candidates, carbon fibre and glass fibre the commonly utilised fibres, together with an epoxy resin are used in most current structural applications. Carbon and glass fibres/epoxy composites are naturally brittle and usually exhibit a linear elastic response up to failure without any plastic deformation. 
Impact damage is always considered as one of the major concerns in FRP structures, as accidental impact from dropped tools, hail stone and bird strike frequently cause damage such as fibre breakage, matrix cracking and delamination [1]. This could lead to the significant decrease of material strength and stiffness particularly under compressive buckling load and hence severely reducing structural integrity and stability. Because of this behaviour, composite structures are susceptible to impact damage problems and they have to satisfy specific certification procedures. For example in aerospace vehicles, the composite structures should be certified at low/high velocity impact from runway debris and bird strike. On the other hand the composite materials can also be designed as an energy absorption component and they are of interest for light weight energy absorbing structural elements if they are suitably designed to trigger failure by delamination and progressive compression crushing $[2,4]$. For the safety reasons in the above applications, it is important to fully understand the mechanisms of delamination, energy absorption and failure in the laminated FRP composites, and to have predictive design tools for simulating the response of the laminated composite structures under impact loading.

The current work is based on the application of explicit finite element software for modelling the behaviour of laminated composite plates under low velocity impact loading and it explores the impact, post impact and failure of these structures. Three models, namely thick shell elements with cohesive interface, solid elements with cohesive interface, and thin shell elements with tiebreak contact, were all developed in the explicit nonlinear finite element code LSDYNA. Correlation of the results from the three models will be investigated by comparing them with the experimental results from literature [5]. The numerical results are utilized in providing guidelines for modelling and impact simulation of FRP laminated composites, and recommendations are provided in terms of modelling and simulation parameters such as element size, number of shell sub-laminates, and contact stiffness scale factors.

\section{Material models}

In this paper all the plates are made from 24 plies of carbon fibre-reinforced composite with stacking sequence of $[-45 / 0 / 45 / 90]_{3 s}$. The carbon fibre-reinforced material properties are $\rho=1.62 \mathrm{~g} / \mathrm{cm}^{3}, E_{11}=153 \mathrm{GPa}$, $E_{22}=10.3 \mathrm{GPa}, G_{12}=5.2 \mathrm{GPa}, X_{t}=2540 \mathrm{MPa}$, $X_{c}=1500 \mathrm{MPa}, Y_{t}=82 \mathrm{MPa}, Y_{c}=236 \mathrm{MPa}$, $S_{C}=90 \mathrm{MPa}$, DFAILT $=0.017$, DFAILC $=0.0135$, DFAILM $=0.1$, DFAILS $=0.03$ and $v_{12}=0.3$ [5]. In all the models, material of impactor was set as MAT20 (MAT_RIGID) and the impactor was constrained in $X, Z$ displacement and all rotations but it was free to move in $Y$ direction.

Damage evolution during the impact of the composite plate was modelled using MAT_ENHANCED_COMPOSITE _DAMAGE (MAT54) and MAT_COMPOSITE_DAMAGE (MAT22), which both based on Chang-Chang failure criteria [6-8].

The Chang-Chang failure criteria for MAT54 and MAT22 follow these conditions:

- tensile fibre failure mode:

$$
\begin{aligned}
& \sigma_{a a}>0 \text { then } \\
& e_{f}^{2}=\left(\frac{\sigma_{a a}}{X_{t}}\right)^{2}+\beta\left(\frac{\sigma_{a b}}{S_{c}}\right)-1\left\{\frac{\geq 0 \text { failed }}{<0 \text { elastic }}\right.
\end{aligned}
$$

after failure $E_{a}=E_{b}=G_{b a}=v_{a b}=v_{b a}=0$;

- compressive fibre failure mode:

$$
\begin{aligned}
\sigma_{a a} & <0 \text { then } \\
e_{c}^{2} & =\left(\frac{\sigma_{a a}}{X_{c}}\right)^{2}-1\left\{\frac{\geq 0 \text { failed }}{<0 \text { elastic }}\right.
\end{aligned}
$$

after failure $E_{a}=v_{b a}=v_{a b}=0$;

- tensile matrix failure mode:

$$
\begin{aligned}
\sigma_{b b} & >0 \text { then } \\
e_{m}^{2} & =\left(\frac{\sigma_{b b}}{Y_{t}}\right)^{2}+\left(\frac{\sigma_{a b}}{S_{c}}\right)^{2}-1\left\{\frac{\geq 0 \text { failed }}{<0 \text { elastic }}\right.
\end{aligned}
$$

after failure $E_{b}=v_{b a}=0 \rightarrow G_{a b}=0$;

- compressive matrix mode:

$$
\begin{aligned}
\sigma_{b b}< & 0 \text { then } \\
e_{d}^{2}= & \left(\frac{\sigma_{b b}}{2 S_{c}}\right)^{2}+\left\lceil\left(\frac{Y_{c}}{2 S_{c}}\right)^{2}-1\right] \frac{\sigma_{b b}}{Y_{c}} \\
& +\left(\frac{\sigma_{a b}}{S_{c}}\right)^{2}-1\left\{\frac{\geq 0 \text { failed }}{<0 \text { elastic }}\right.
\end{aligned}
$$

after failure $E_{b}=v_{b a}=v_{a b}=0 \rightarrow G_{a b}=0$;

where in Equations 1-4 the subscript "a" is fibre direction and the subscript " $b$ " is transverse direction (normal to fibre).

In MAT54 failure can occur in any of the following four cases:

- If DFAILT (maximum strain for fibre tension) is zero, failure occurs in the tensile fibre mode if the ChangChang failure criterion is satisfied. 
- If DFAILT is greater than zero, failure occurs if the tensile strain is greater than DFAILT or less than DFAILC (maximum strain for fibre compression).

- If EFS (effective failure strain) is greater than zero, failure occurs if the effective strain is greater than EFS.

- If TFAIL (time step size criteria for element deletion) is greater than zero, failure occurs according to the element time step.

After failure occurs in the entire composite layers (throughthickness integration points), the element is deleted and the attached elements to the deleted element become "crashfront" elements. In this case their strength can be reduced by SOFT parameter with TFAIL greater than zero [9].

\section{Delamination in laminated com- posite structures}

One of the main sources of damage in laminated composite structures is delamination, separation of the plies in the low resistance thin resin-rich interface between adjacent layers particularly under compressive loading, impacts or free-edge stresses. This is more of a problem when there is lack of any reinforcement in the thickness direction. Delamination occurs when interlaminar stresses in the boundary layer of the plies increases as a result of applied transverse loading and causing the layers to debond. Other causes of delamination are the existence of contaminated fibres during the manufacturing process, insufficient wetting of fibres, curing shrinkage of the resin, and outof-plane impact. The existence of high stress gradients near geometric discontinuities in composite structures such as holes, cut-outs, flanges, ply drop-offs, stiffener terminations, bonded and bolted joints promote delamination initiation. There is nondestructive evidence that shows a large extent of delaminations occurring between individual plies under impact loading [5]. The presence and growth of delamination in laminates significantly reduces the overall buckling strength of a structure while delamination grows rapidly in the post-buckling region. Delamination is an important energy absorption mechanism and it also reduces the load-carrying capacity in bending and the fatigue life of the structures.

\subsection{Cohesive zone element}

In the absence of any nonlinearity, there are many techniques in linear elastic fracture mechanics (LEFM), such as the virtual crack extension [10], the J-integral [11], the virtual crack closure (VCC) $[12,13]$, and the stiffness derivative [14] based on the Griffith criteria [15] that the crack propagates when the associated energy release rate at the crack tip is greater than or equal to the critical value of fracture toughness that can be used to predict the delamination in composite structures. However, these methods cannot be used to predict the initiation of the delamination and, therefore, they are restricted to problems in which the initial position of the crack is known [16].

Other methodologies have been developed to model the mechanical behaviour of the interface on the basis of damage mechanics and/or softening plasticity combined with an indirect introduction of fracture mechanics [17-24]. One of these methodologies, which may be considered to stem from the work of Hillerborg et al. [17], includes the Cohesive Zone Model (CZM) [18-22]. The viewpoint from which cohesive zone models originate regards fracture as a gradual phenomenon in which separation takes place across an extended crack 'tip', or cohesive zone, and is resisted by cohesive tractions. Thus cohesive zone elements do not represent any physical material, but describe the cohesive forces which occur when material elements (such as grains) are being pulled apart. In relation to the simulation of delamination, the method has often been applied in conjunction with interface elements [23]. The origin of CZM in ductile materials dates back to Dugdale [25], who used it for elastoplastic fracture in ductile metals. In his model the cohesive strength is assumed to be equal to the yield strength and to be constant along the cohesive zone. Later, more realistic cohesive models were introduced [18-22]. In these models, the initiation of crack growth occurs when the critical separation is attained. In contrast to the Dugdale model, these models have an initial elastic behaviour followed by a region of progressive damage (softening part), where the magnitude of the traction decays during decohesion. Williams and Hadavinia [26] presented analytical solutions for cohesive zone models and calculated correction factors for composite DCB specimens and their extension to peel testing. CZM are now widely used to describe local fracture processes [27-29].

The CZM, also known as Embedded Process Zone (EPZ) [21], Damage Zone Model (DZM), or Fictitious Crack Model (FCM) [24], is used to model crack growth at the interfaces. The cohesive zone is a surface in a bulk material where displacement discontinuities occur. Thus, continuum is enhanced with discontinuities in the form of displacement jumps. The fracture process is governed by two parameters: intrinsic toughness of the interface and the cohesive strength, $\sigma_{\max }[18-23]$, generally related by the cohesive constitutive law

$$
\sigma=\sigma_{\max } f(\lambda)
$$


a) Bilinear

c) Parabolic

Figure 1. Different forms of the traction-separation law.
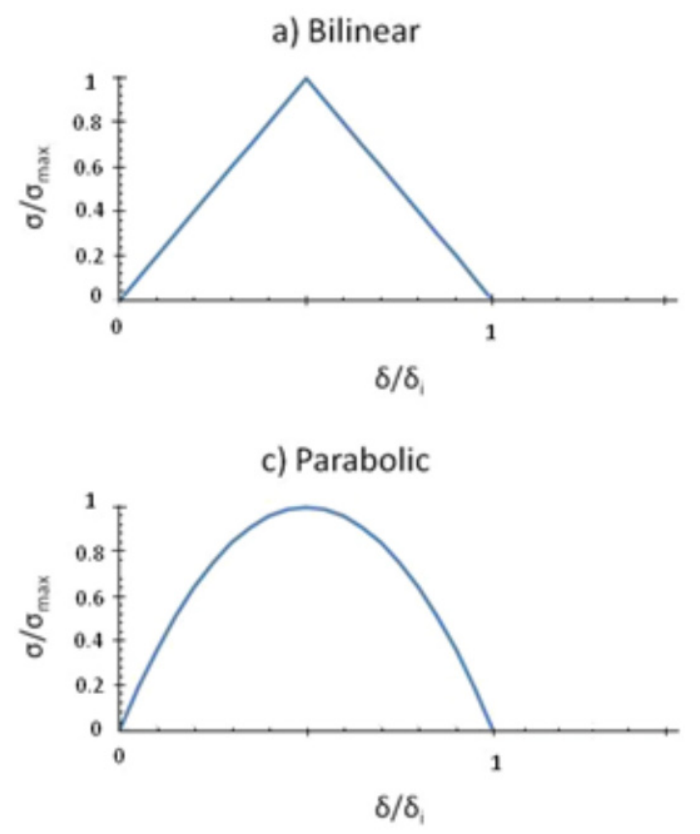

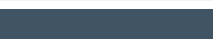
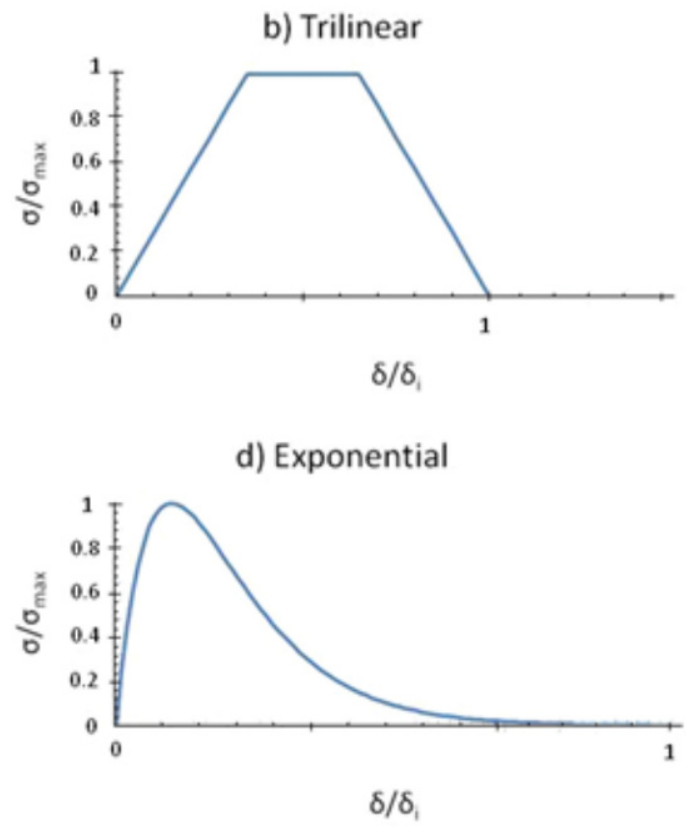

where $\lambda=\delta / \delta_{n c}$ and $\delta_{n c}$ is the critical crack tip opening displacement and $f(\lambda)$ is a dimensionless function describing the shape of the cohesive law (see Figure 1). This is introduced by a variation in the cohesive stresses with the interfacial opening/shearing displacement along the localized fracture process zone, a small zone in front of the crack tip, in which small-scale yielding (SSY), microcracking or void growth, and coalescence take place. In the traction-separation law, generally tractions increase reversibly until it reach a maximum, and then approach zero in the softening section with increasing separation. The area under the traction-separation relationship is the fracture energy or work of separation. There are plenty of traction-separation laws in the literature among them: Barenblatt [29], Dugdale [25], Needleman [18], Rice and Wang [31], Tvergaard and Hutchinson [24], Xu and Needleman [32], Camacho and Ortiz [22], Blackman et al. [28] and Geubelle and Baylor [33]. These traction-separation laws can be classified as bilinear, trapezoidal (or trilinear), parabolic, and exponential as shown in Figure 1.

In FRP composites due to the damage zone around the crack tip, failure is mainly governed by energy-based criteria rather than critical stress or strain. As in the CZM the fracture energy is incorporated as a basic parameter in its procedure, this makes it an appropriate methodology to predict the global failure at various scales of composite debonding such as decohesion between the matrix and fibres and delamination between the laminae by introducing the local fracture parameters. Parameters characterizing traction-separation have been determined by fitting model predictions to a selected set of experiments, thereby providing a calibration against the fracture process at the smallest relevant scale [34, 35].

The FE analysis will be carried out on a bench mark of a carbon fibre-reinforced composite plate with lay-up $[-45 / 0 / 45 / 90]_{3 s}$ with dimensions of $300 \mathrm{~mm} \times 150 \mathrm{~mm} \times$ $2.7 \mathrm{~mm}(\mathrm{~L} \times \mathrm{W} \times \mathrm{t})$. The impactor was modelled as a sphere with a diameter of $25.4 \mathrm{~mm}$ and a mass of $1.85 \mathrm{~kg}$. The plate and impactor are meshed as shown in Figure 2. From previous studies on mesh sensitivity analysis [1] the element size in FE model was set to $5 \mathrm{~mm}$ and impactor meshed freely. The low velocity impact experiments on this plate are reported on [5].

\subsection{Modelling delamination with $\mathrm{CZ}$ elements}

There are many material models which can be used to simulate interface debonding in LS-DYNA. These are material types: MAT138 ("MAT_COHESIVE_MIXED_MODE), MAT184 ("MAT_COHESIVE_ELASTIC), MAT185 ("MAT_COHESIVE_TH), MAT186 ("MAT_COHESIVE _GENERAL), and MAT240 ("MAT_COHESIVE_MIXED _MODE_ELASTOPLASTIC_RATE) which its traction separation law is similar to MAT185 but it further considers the effect of plasticity and rate dependency and tiebreak contacts ("CONTACT_AUTOMATIC_ONE_WAY_SURFACE_TO_SURFACE_TIEBREAK). 


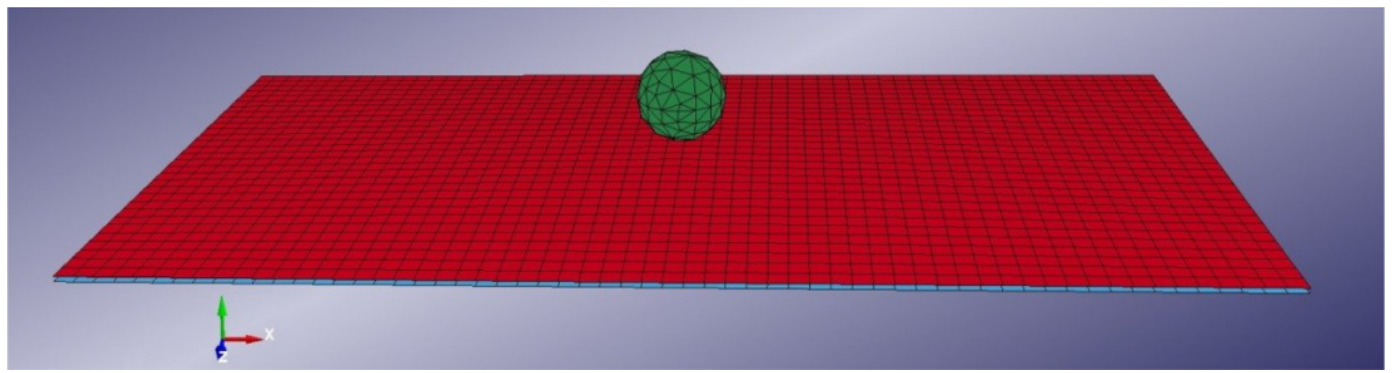

Figure 2. LS-DYNA model of a laminated composite plate and impactor.
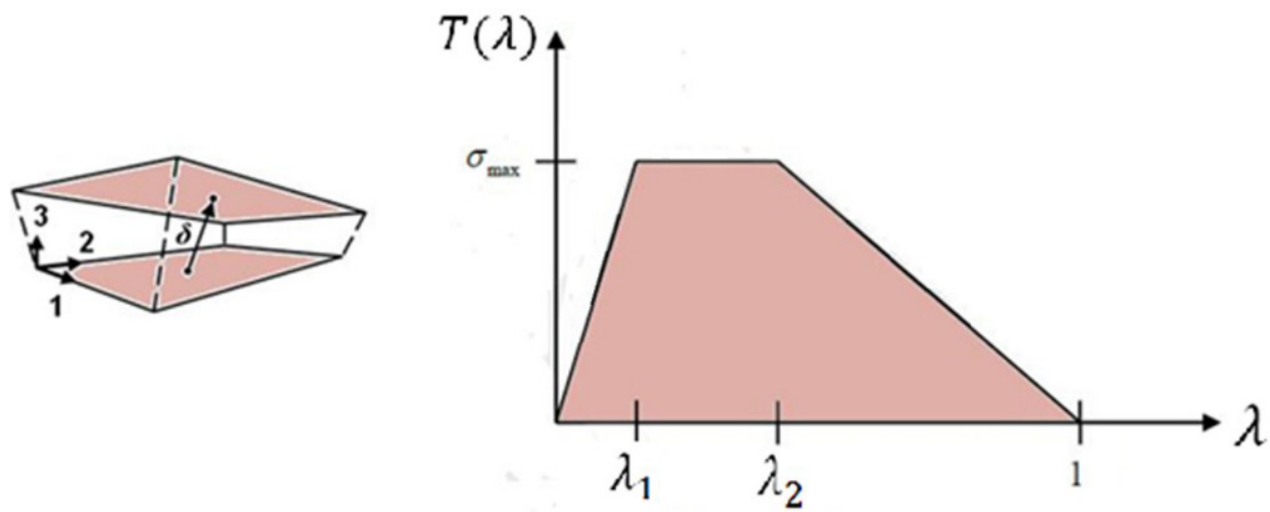

Figure 3. Trilinear traction-separation law for material type MAT185 [8].

It has been shown that the shapes of the tractionseparation law have a secondary influence on the delamination behaviour for quasi-static cases [26]. However under impact loading, the abrupt change of stiffness in bilinear cohesive law shown in Figure 1a make some instability in the solution. Therefore in the current work material model MAT185 (*MAT_COHESIVE_TH) is used for delamination at the interface between the plies which is based on a trilinear traction-separation law as shown in Figure 3.

In MAT185 a dimensionless parameter, $\lambda$, representation of an equivalent relative separation in three modes of opening, shearing and tearing $\left(\delta_{3}, \delta_{1}, \delta_{2}\right)$ directions is calculated by:

$$
\lambda=\sqrt{\left(\frac{\delta_{1}}{T L S}\right)^{2}+\left(\frac{\delta_{2}}{T L S}\right)^{2}+\left(\frac{<\delta_{3}>}{N L S}\right)^{2}}
$$

where the Macaulay bracket is zero for negative value (compression) and NLS and TLS are critical separations at the interface in normal and tangential directions, respectively.
The trilinear tractions-separation law is:

$$
T(\lambda)= \begin{cases}\sigma_{\max } \frac{\lambda}{\lambda_{1}} & \lambda<\lambda_{1} \\ \sigma_{\max } & \lambda_{1}<\lambda<\lambda_{2} \\ \sigma_{\max } \frac{1-\lambda}{1-\lambda_{2}} & \lambda_{2}<\lambda<1\end{cases}
$$

where the traction drop to zero at $\lambda=1 . \lambda_{1}$ and $\lambda_{2}$ are scaled distance to the beginning of peak traction and to the beginning of softening, respectively, see Figure 3 .

Based on the relative displacement, a potential is defined

$$
\phi\left(\delta_{1}, \delta_{2}, \delta_{3}\right)=N L S \cdot \int_{0}^{\lambda} T(\hat{\lambda}) d \hat{\lambda}
$$

The components of tangential and normal tractions acting on the interface can be found from:

$$
\begin{aligned}
T_{1,2} & =\frac{\partial \phi}{\partial \delta_{1,2}}=\frac{T(\lambda)}{\lambda} \cdot \frac{\delta_{1,2}}{T L S} \cdot \frac{N L S}{T L S} \\
T_{3} & =\frac{\partial \phi}{\partial \delta_{3}}=\frac{T(\lambda)}{\lambda} \cdot \frac{\delta_{3}}{N L S}
\end{aligned}
$$

In case of compression $\left(\delta_{3}<0\right)$, penetration is avoided by setting

$$
T_{3}=P S \cdot \delta_{3}
$$



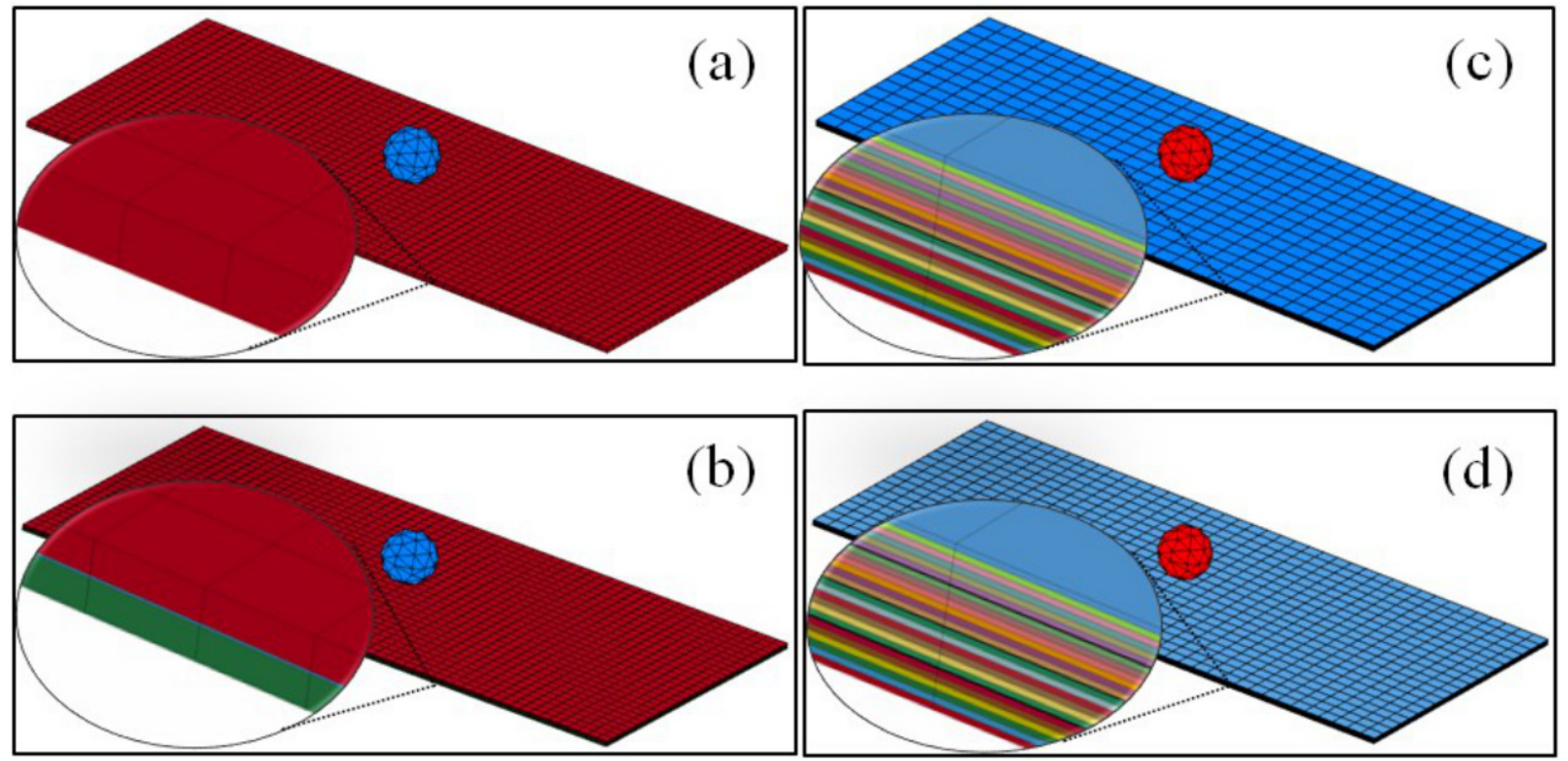

Figure 4. FE models of impacted laminated composite plate (a) one TSHELL; (b) two TSHELL sublaminates with a CZ layer; (c) SL-CZ-SL and (d) SL-CZ-SL-CZ-SL-CZ-SL (black lines between layers in (c) and (d) are the cohesive layer).

where penetration stiffness multiplier $(P S)$ is calculated from:

$$
P S=\frac{S T F S F \cdot \sigma_{\max }}{N L S \cdot \lambda_{1}}
$$

\subsection{Modelling plates with thick shell and solid elements}

Laminated composite materials can be modelled in three different ways in LS-DYNA platform. The first method is using thin shell elements (SHELL163) with tiebreak contact for delamination (will be covered in Section 4). The second method is to employ multilayer thick shell (TSHELL) formulations by setting the number of integration points equal to the number of layers and angles of each layer (Figure $4 \mathrm{a}$ and $4 \mathrm{~b}$ ). The delamination in the thick shell will be modelled by cohesive zone elements. The last method is to use solid layers (SL) with their material angle together with cohesive layers between the solid layers (Figure $4 c$ and $4 d$ ).

In most FEA studies, thick composite structures are modelled by shell elements which produce inaccurate results in the transverse direction. Therefore, a 3D modelling for thick composite structures is preferable as the through thickness stress variation can be captured accurately. However, the use of solid elements is restricted as by using even one layer of solid elements in each layer, the size of the model becomes huge which dramatically increases the solution time. Another alternative is to use a multi-layered solid element which is now implemented in LS-DYNA code (Version 971 R4 and later) as a thick shell (TSHELL) showing excellent efficiency of CPU solution time. The representation of several plies in one solid element across the thickness will be explored in this part.

8-node layered solid elements in LS-DYNA can be accessed by using thick shell element formulation 5, which uses one integration point per layer and any number of layers similar to thin shell elements (SHELL163). Thick shell element formulation 5 together with *PART_COMPOSITE or "INTEGRATION_SHELL commands are used to define thickness and fibre direction for each integration point to model properly a layered composite.

In the thick shell models; one TSHELL with 24 integration points through the thickness (Figure 4a) and 2 TSHELL with one cohesive layer (MAT185) were used for delamination (Figure $4 \mathrm{~b}$ ). The plate was also modelled by solid elements in each layer setting their material angle together with cohesive layers (MAT185) between these solid layers (Figure $4 c$ and $4 d$ ). In the modelling with solid layer (SL), two different models analysed; 12SL-CZ-12SL (Figure 4c) and 6SL-CZ-6SL-CZ-6SL-CZ-6SL (Figure 4d).

The material model of impactor was set as MAT20 (MAT_RIGID) and the impactor was constrained in X, Z displacement and all rotations but free to move in $Y$ direction. The impactor velocity was $6.5 \mathrm{~m} / \mathrm{s}$ corresponding to $39 \mathrm{~J}$. In all the modelling the delamination fracture toughness in mode I is set at $G_{\mid C}=230 \mathrm{~J} / \mathrm{m}^{2}$ and for mode $\|$ at $G_{\| C}=$ $650 \mathrm{~J} / \mathrm{m}^{2}$. 


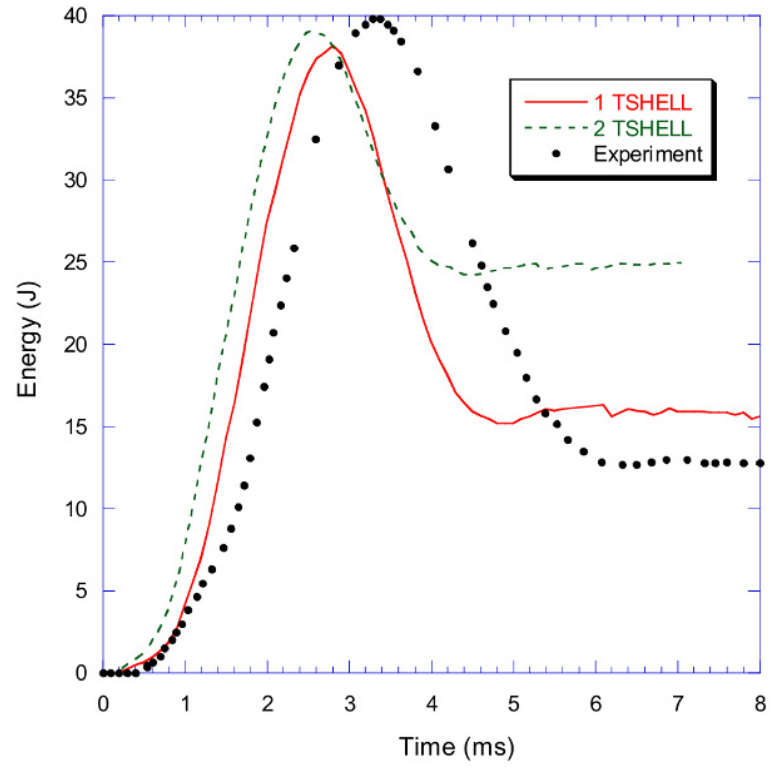

(a)

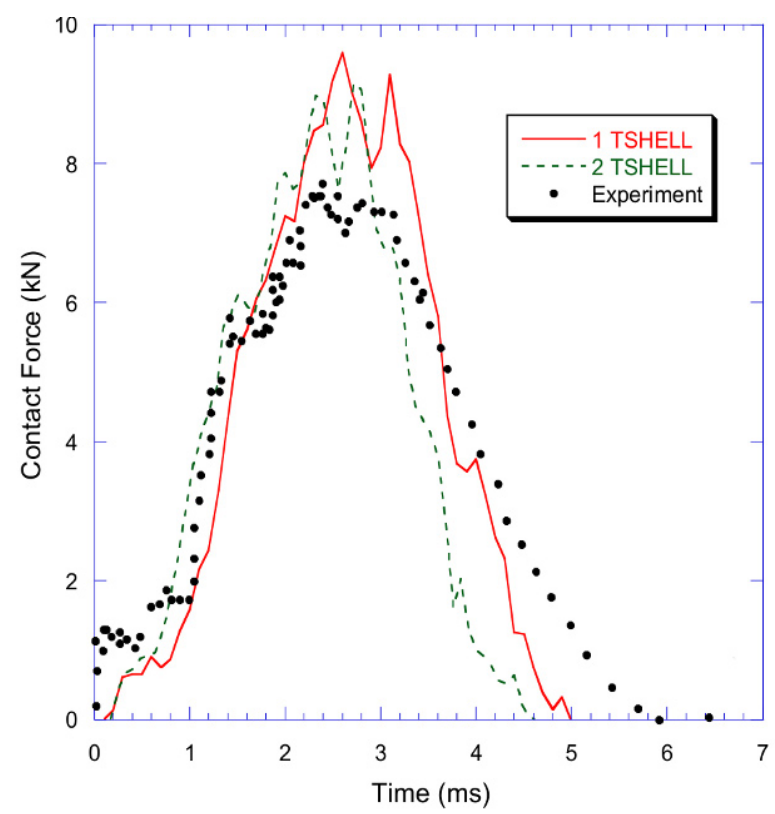

(b)

Figure 5. Impacted laminated plate modelled with TSHELL elements (a) internal energy and (b) contact force. Experiment from [9].

Figure 5a compares the internal energy of composite plate during the impact for thick shell (TSHELL) models. The internal energy of FE model with one TSHELL gives a closer energy absorption results which is $15 \%$ higher than the experiment with a lag time in the peak energy. Increasing the number of TSHELL sublaminates through the thickness with a CZ bonding layer between them with a thickness of $0.01 \mathrm{~mm}$ (MAT185) resulted in slightly higher peak energy levels and $12 \%$ higher absorbed energy, but during the rising energy it matches more closely to the experiment. This extra energy absorption occurred because of delamination between the TSHELL layers.

The effect of the cohesive bonding layer on contact force is demonstrated in Figure 5b. The 2 TSHELL model again match the experiment more closely. The hourglass energy in the impact simulation was negligible, at around $1 \%$ of the total energy.

The impact response at the beginning of contact time for both one and two TSHELL models is similar. The composite plate modelled with 2 TSHELL sublaminates and a cohesive bonding layer behaves slightly better than one TSHELL model.

Both thick shell and solid elements were modelled with MAT22 ("MAT_COMPOSITE_DAMAGE) in LS-DYNA. Figure 6 shows the tensile matrix mode failure index, em, for $1^{\text {st }}, 2^{\text {nd }}, 7^{\text {th }}$ and $12^{\text {th }}$ integration points and their symmetrical layers at peak impact load around $2.9 \mathrm{~ms}$ for one TSHELL model.. The maximum damage occurred in plies with $90^{\circ}$ orientation and the least damage occurred in plies $0^{\circ}$ orientation.

The plate was also modelled by solid elements in each layer with cohesive layers with a thickness of $0.01 \mathrm{~mm}$ (MAT185) between the solid layers. In the modelling with solid layers (SL), two different models analysed: 12SL-CZ12SL and 6SL-CZ-6SL-CZ-6SL-CZ-6SL.

Figure 7a compares the internal energy of composite plate during the impact for 12SL-CZ-12SL and 6SL-CZ-6SLCZ-6SL-CZ-6SL solid models. The energy absorption of 12SL-CZ-12SL model is nearly the same as experiment with a lag time in the peak energy. Similar to the TSHELL models, increasing the number of $\mathrm{CZ}$ layers caused an increase of around $20 \%$ on the absorbed energy. The effect of cohesive bonding layers on contact force is shown in Figure 7b. The estimate of contact force in both models are similar with small time lag in the prediction of the peak contact force.

\section{Impact modelling of composite structures using tiebreak contact}

In laminated composite plate the delamination can also be modelled using tiebreak delamination contact between sublaminates in the LS-DYNA. Basically tiebreak contacts are used as adhesive to bond the sublaminates in the LS-DYNA models. Tiebreak contacts are active for nodes which are initially in contact. Normal and shear failure strength must be defined for tiebreak contact to check the bond failure. Tiebreak contact acts the same way as 

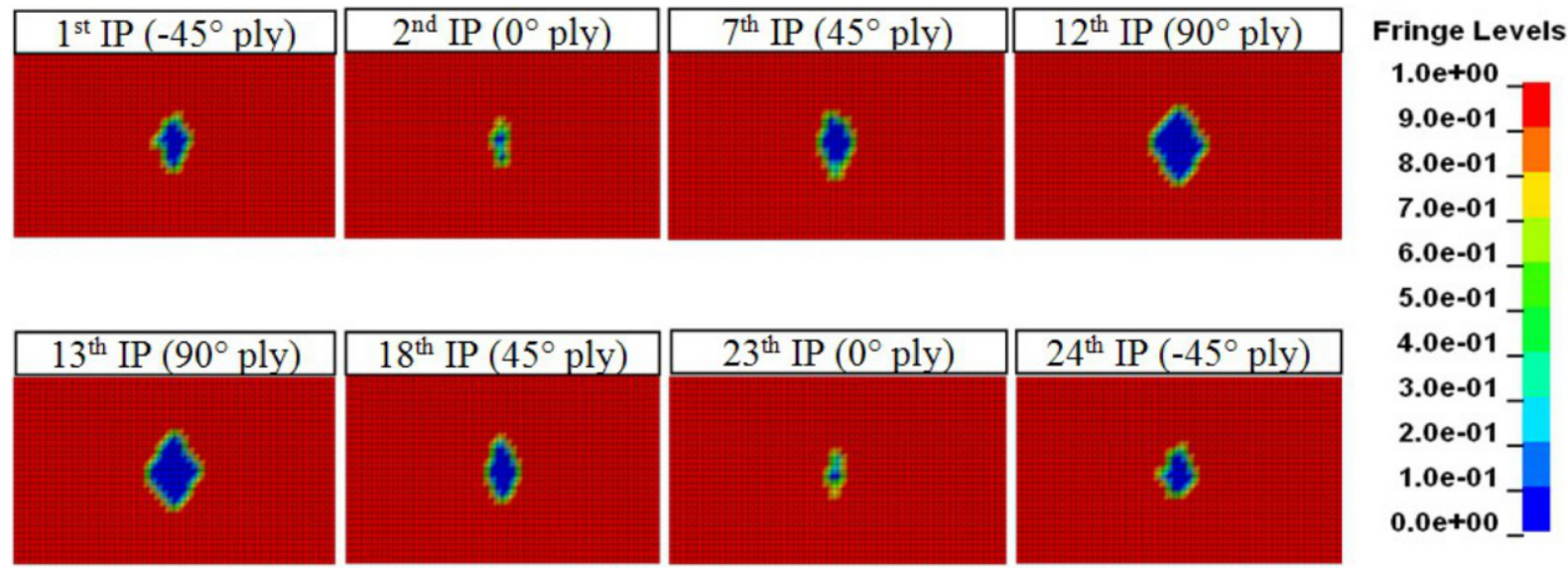

Figure 6. Contour of tensile matrix mode failure $\left(e_{m}\right)$ at different integration point in one TSHELL model at peak load $(2.9 \mathrm{~ms})$. IP $=$ integration point.

other common contacts under compressive load. During the loading, the damage of the material is a linear function of the distance between the two points which are initially in contact. When the critical opening is reached, the contact will be broken and the sublaminates are converted into two separate surfaces with regular surface to surface contact between them to prevent penetrations.

Under tensile load, tiebreak allows the separation of the surfaces and ultimately the failure of the tied surfaces will occur under the following failure criterion:

$$
\left(\frac{\left|\sigma_{n}\right|}{N F L S}\right)^{2}+\left(\frac{\left|\sigma_{S}\right|}{S F L S}\right)^{2} \geq 1
$$

where NFLS is tensile failure strength and SFLS is shear failure strength of the adhesive.

An adhesive material with the properties of NFLS = $56 \mathrm{MPa}$ and SFLS $=44 \mathrm{MPa}$ has been chosen. These are typical properties for epoxy adhesives usually used in structural applications.

The effect of the number of sublaminate on the impact behaviour of the composite plate with 24-plies in 1 shell and 12-plies in 2 sublaminates is investigated using multilayered SHELL163. Figure 8 compares FE and experimental results in [5]. This verifies that impact analysis can be modelled accurately with thin shell element (SHELL163) and tiebreak contacts. It is evident that both 1 thin shell and 2 thin shells models matching with experiment. However, 2 thin shells model has a more stable contact force results.

Further studies were carried out on a stiffened composite plate substructure as shown in Figure 9 to investigate the effect of contact penalty stiffness SFS and SFM, SOFT option in optional card of CONTACT command and OPTION in one of the mandatory cards in tiebreak contacts. 2-shell model with tiebreak contact was selected for further studies. The material properties of the plates are as before. The impactor density set at $\rho=1.794 \mathrm{~g} / \mathrm{cm}^{3}$ and velocity at $6.5 \mathrm{~m} / \mathrm{s}$ corresponding to $1000 \mathrm{~J}$.

Different contact definitions are needed between components in the model. CONTACT_AUTOMATIC_ SURFACE_TO_SURFACE are used between impactor and plates and between components of plate (i.e. angled plate and top plate components of the stiffened plate). CONTACT_AUTOMATIC_ONE_WAY_SURFACE_TO_SURFACE_TIEBREAK and CONTACT_AUTOMATIC_SURFACE_TO_SURFACE_TIEBREAK were used between sublaminates of the composite plate in all models. Different OPTION in contact card was used for simulation of crack propagation based on the cohesive zone model, implemented in LS-DYNA as a delamination contact.

\subsection{Tiebreak contact parameters}

Treatments of sliding and impact along interfaces are very critical in simulating the correct load transfer between different components in an analysis. The generated contact forces influence the acceleration of the structure. Contact algorithms employed in LS-DYNA finite element codes divide the nodes of bodies involved in contact into slave and master nodes. Then each slave node is checked for penetration against master element face. Therefore using a robust contact algorithm that can efficiently track and generate appropriate forces to the slave nodes without generating spurious results is very important. Three different methods, the kinematic constraint method, the penalty method and the distributed method, are implemented in LS-DYNA. A brief discussion of the three methods with merits and demerits is described below. 


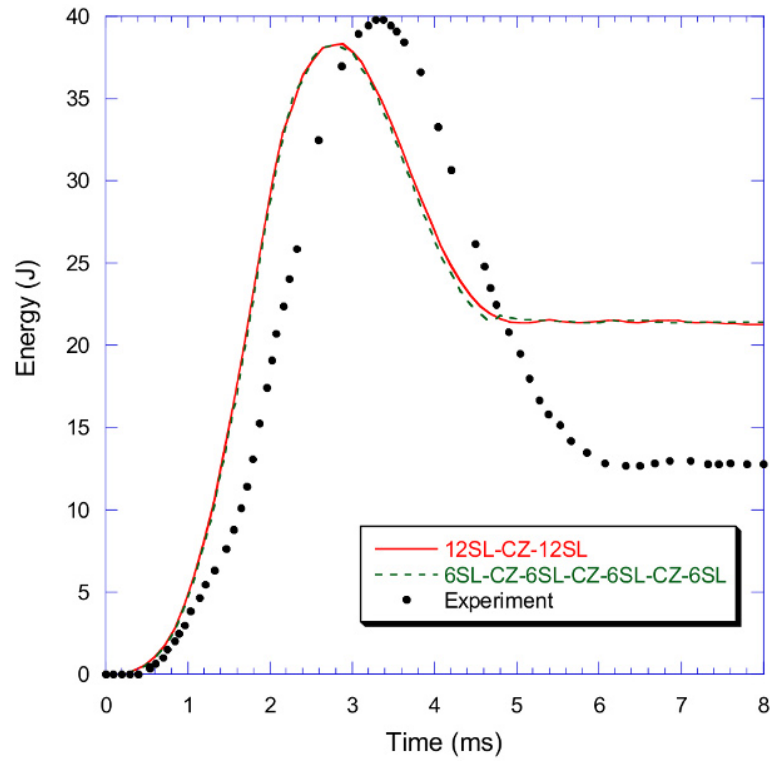

(a)

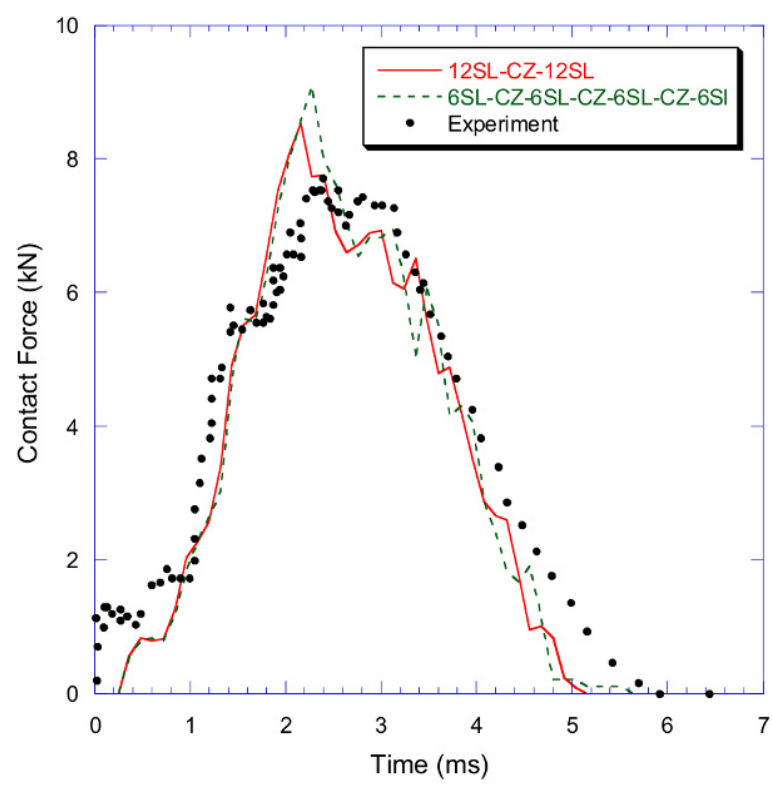

(b)

Figure 7. Impact of laminated plate modelled with solid elements (a) internal energy and (b) contact force. Experiment from [5].

The first method is the kinematic constraint method which uses the impact and release conditions of Hughes et al. [36]. Constraints are imposed on the global equations by a transformation of the nodal displacement components of the slave nodes along the contact interface. This transformation has the effect of eliminating the normal degree of freedom of nodes. Since computational efficiency of the

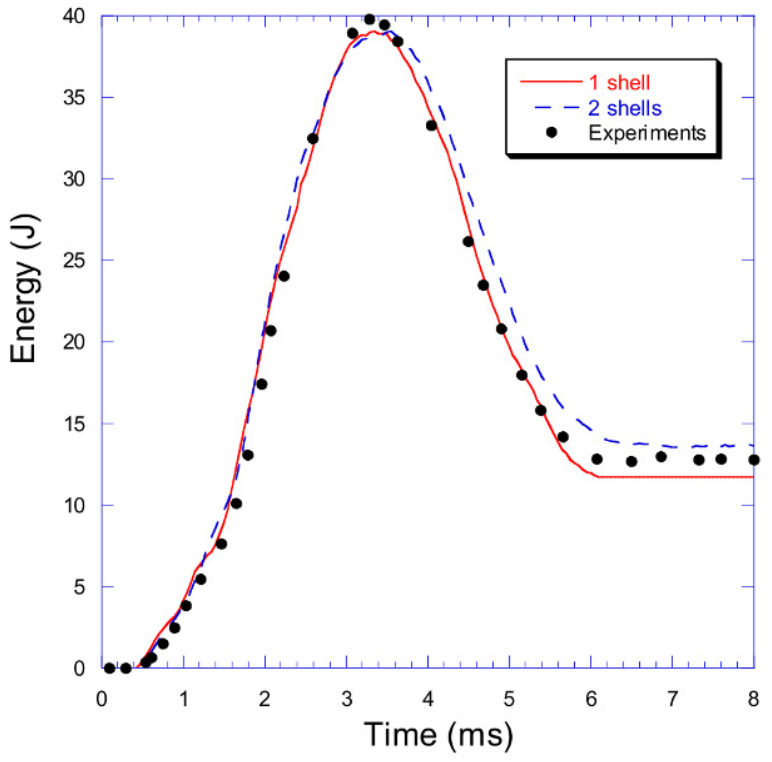

(a)

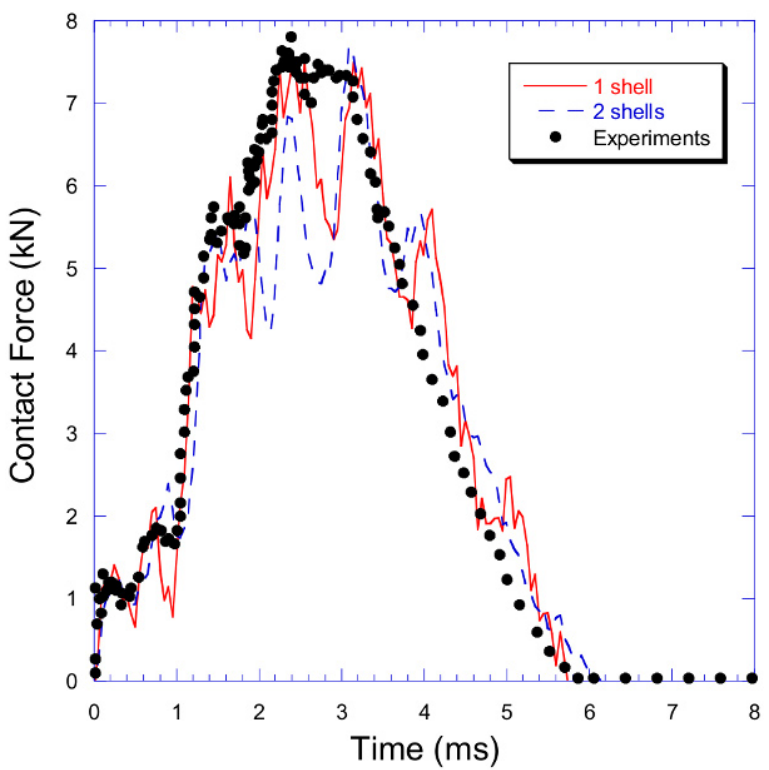

(b)

Figure 8. Internal Energy and Contact Force for impact analysis with using thin shell (SHELL163) and Tiebreak contacts. Experiment from [5].

explicit time integration needs to be preserved, the mass is lumped to the extent that only the global degrees of freedom of each master node are coupled. Impact and release conditions are imposed to insure momentum conservation. This method has the advantage when two materials in contact have very different material properties. The nodes are constrained to stay on or very close to the surface without 


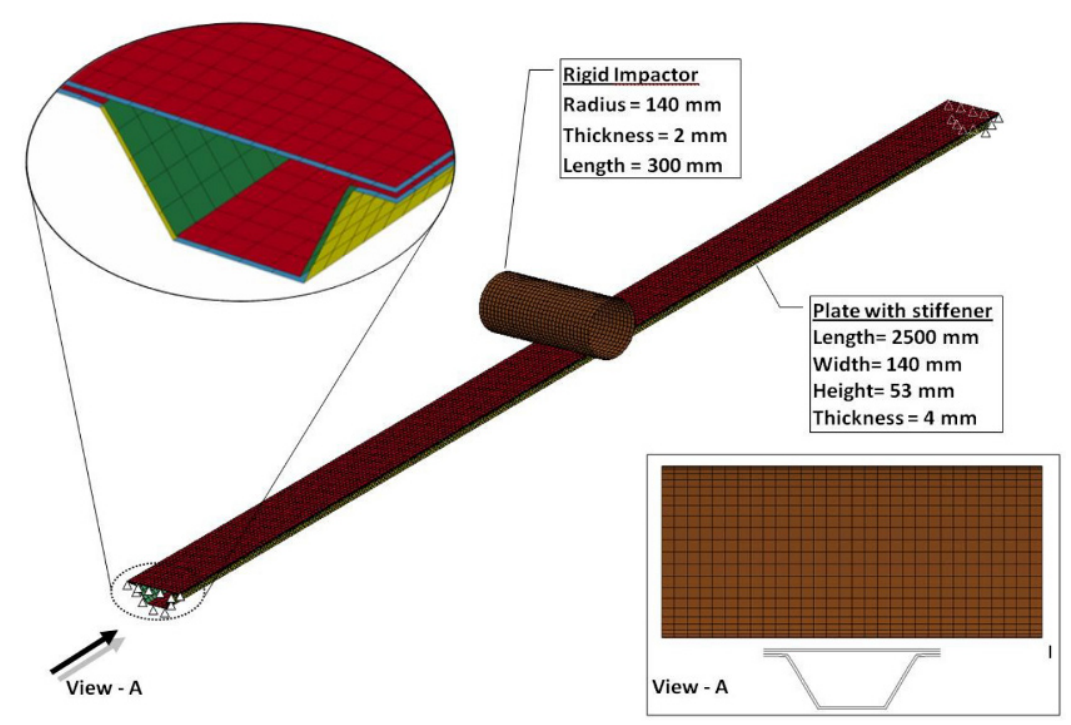

Figure 9. Composite stiffened plate; 12 plies in each sublaminates with stacking sequence of $[-45 / 0 / 45 / 90]_{3 s}$.

causing penetrations due to the difference in the stiffness. However problems arise when the master surface zoning is finer that the slave surface zoning. Certain master nodes can penetrate through the slave surface without resistance and create a kink in the slide line.

The second method is called penalty-based approach. Contact treatment is internally represented by linear springs between the slave nodes and the nearest master segments. The stiffness of these springs determines the force that will be applied to the slave nodes and master nodes. Currently, two different methods are used for calculating the spring stiffness.

In the first way in penalty-based approach SOFT $=0$ is considered in the contact card. This method consists of placing normal interface springs between all penetration nodes and the contact surfaces. With the exception of the spring stiffness matrix, which must be assembled in the global stiffness matrix, the implicit and explicit methods are similar. Momentum is conserved without the necessity of impact and release conditions.

The stiffness of the contact springs are calculated from:

- for segments on solid elements

$$
k=\frac{f_{s} \times A^{2} \times K}{V}
$$

- And for segments on shell elements

$$
k=\frac{f_{s} \times A^{2} \times K}{\text { Minimum Diagonal }}
$$

where $A=$ Area of the contact segment, $K=$ bulk modulus of contacted element, $V=$ Volume and $f_{S}=S L S F A C \times$ $S F S$ for slave element and $f_{s}=S L S F A C \times S F M$ for master elements. SLSFAC is penalty scale factor and set to 0.1 and SFS/SFM is scale factor for slave/master penalty stiffness. This method is the default method and uses the size of the contact segment and its material properties to determine the contact spring stiffness. As this method depends on the material constants and the size of the segments, it works effectively with the same orderof-magnitude material stiffness parameters between the contacting surfaces. In cases where dissimilar materials come into contact, the contact might break down, as the stiffness, which is roughly the minimum of the slave and master stiffness, maybe too small. This frequently happens with soft dense foams in contact with metals. Consequently, for crash analysis SOFT $=0$ is not recommended, unless prior experience shows that no problems occur.

The contact spring stiffness for a contact segment is calculated based on the material properties of the component involved in the contact. If two different materials with varying stiffness such as foam and steel come in contact, the stiffness of the lesser magnitude is taken as the contact stiffness. This causes penetration problems as the force generated by foam is small compared to the force generated by steel. This is overcome by scaling fs until the forces generated by the two materials are in equilibrium. Determining the appropriate value of $f s$ is important for the forces generated to be in equilibrium.

The second way is the soft constraint-based approach which calculates the stiffness of the linear contact springs based on the nodal masses that come into contact and 


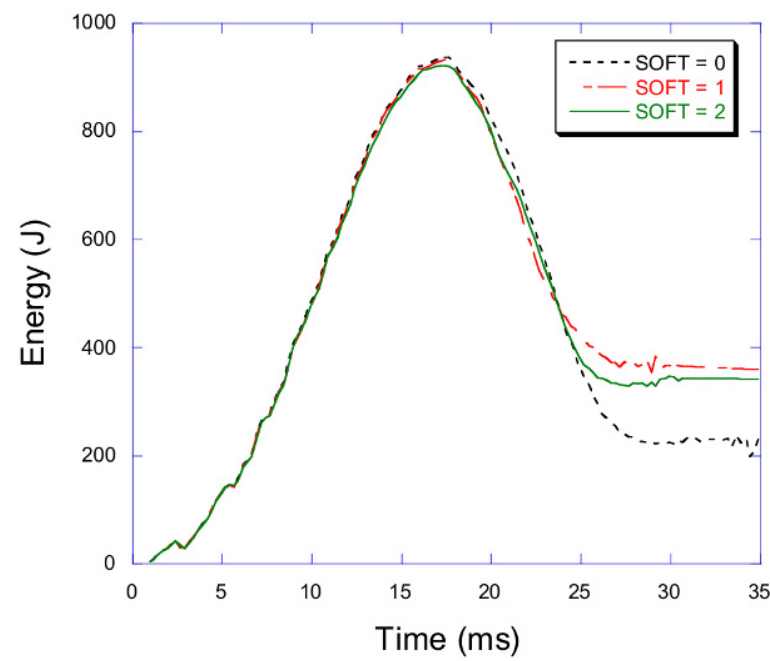

(a)

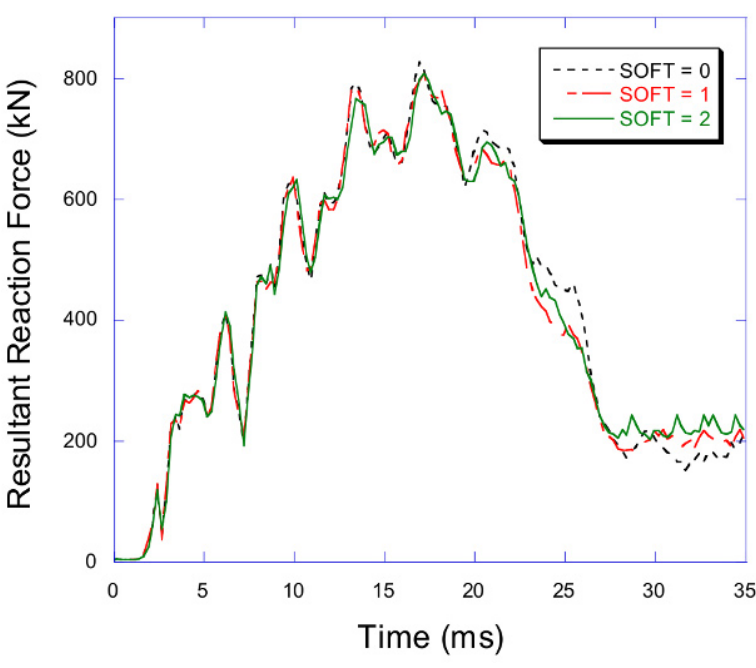

(b)

Figure 10. Comparison of (a) absorbed energy and (b) reaction force from FEA of the impacted substructure with 1 shell (24 integration points) with different SOFT magnitude.

global time step size. The resulting contact stiffness is independent of the material properties and is well suited for treating contact between bodies of dissimilar materials and the stiffness is found by taking the nodal mass divided by the square of the time step size with a scale factor to ensure stability.

$$
k=\operatorname{SOFSCL} \frac{m}{\Delta t^{2}}
$$

where SOFSCL $=0.1$ is the default.

Generally, for the case of metals contacting metals the resulting penalty stiffness for SOFT $=0$ and SOFT $=1$ is similar. The SOFT $=1$ option is recommended for impact analysis where dissimilar materials come into contact. The SOFT $=2$ option uses mass and time step size based penalty stiffness as SOFT $=1$. SOFT $=2$ invokes a segment-based contact algorithm developed by Belytschko et al. [37]. With this algorithm, contact between segments is treated rather than using the usual node-to-segment treatment. When two 4-noded segments come into contact, forces are applied to eight nodes to resist segment penetration. This treatment has the effect of distributing forces more realistically and sometimes is quite effective for very stubborn contact problems.

Parameter SFS and SFM which are in card 3 in "CONTACT (option) are penalty scale factors. SLSFAC in * CONTROL_CONTACT scales the stiffness of all penaltybase contacts, which have the parameter SOFT set equal to 0 or 2 . For $S O F T=1$, the penalty scale factors have no effect. The default values (SFS $=S F M=1.0$ and
SLSFAC $=0.1)$ generally work well for contact between similarly refined meshes of comparable stiffness materials. For contact involving dissimilar mesh sizes and dissimilar material constants, non-default value penalty scale factors may be necessary to avoid the breakdown of contact if $\mathrm{SOFT}=0$.

The last method is the distributed parameter method. This method is derived from TENSOR [38] and HEMP [39] program, which displaces fewer mesh instabilities compared to the nodal constraint algorithm. In this method, one half of the slave element mass of each element in contact is distributed to the covered master surface area. Also, the internal stress in each element determines a pressure distribution for the master surface area that receives that mass. After the distribution of mass and pressure, the acceleration of the master surface is updated.

\subsection{Effect of contact parameters}

The impact model of the stiffened plate with 2 sublaminates (12 integration points in each sublaminate) is chosen as the bench mark to investigate the influences of SOFT parameter magnitude in *CONTACT card. Three different values of SOFT parameter selected are SOFT $=0, \mathrm{SOFT}=1$ and SOFT $=2$ for optional card A in "CONTACT command. In this case only one contact between the plate and the impactor was defined which is CONTACT_AUTOMATIC_SURFACE_TO_SURFACE.

The internal energy and contact forces for different SOFT values are compared in Figure 10. The figure shows that there is no significant change in absorbed energy with 


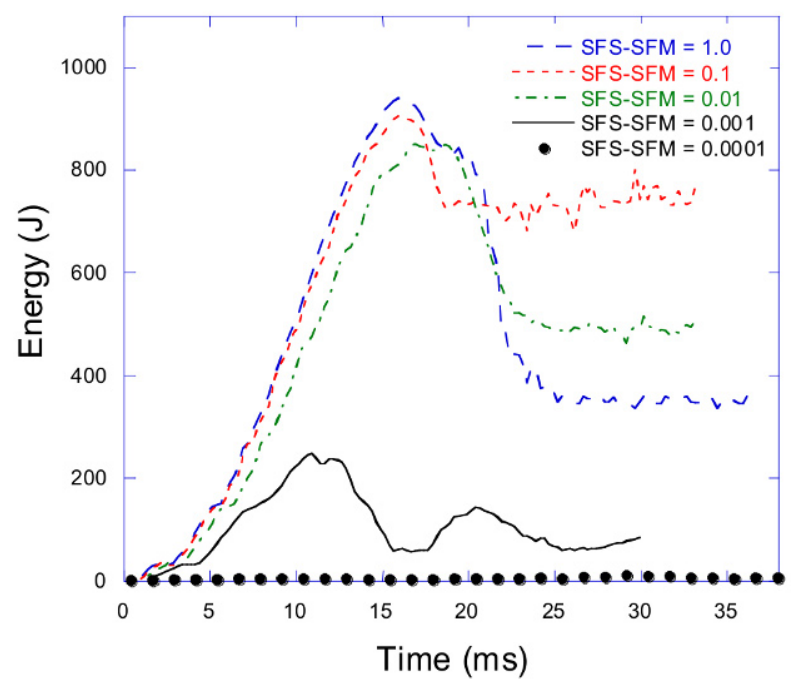

(a)

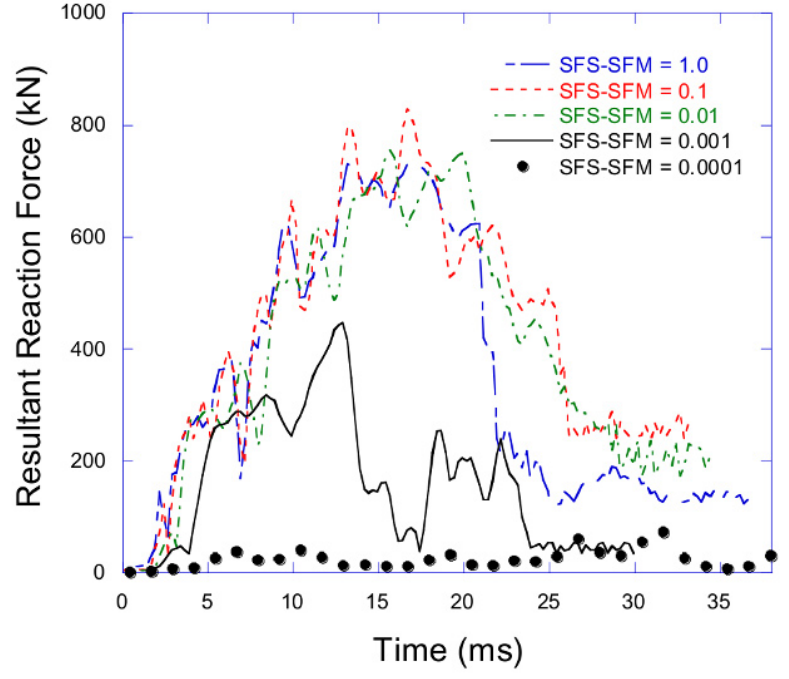

(b)

Figure 11. Comparison of absorbed energy of the impacted plate modelled with 1 shell (24 integration points) with different SFS/SFM values.
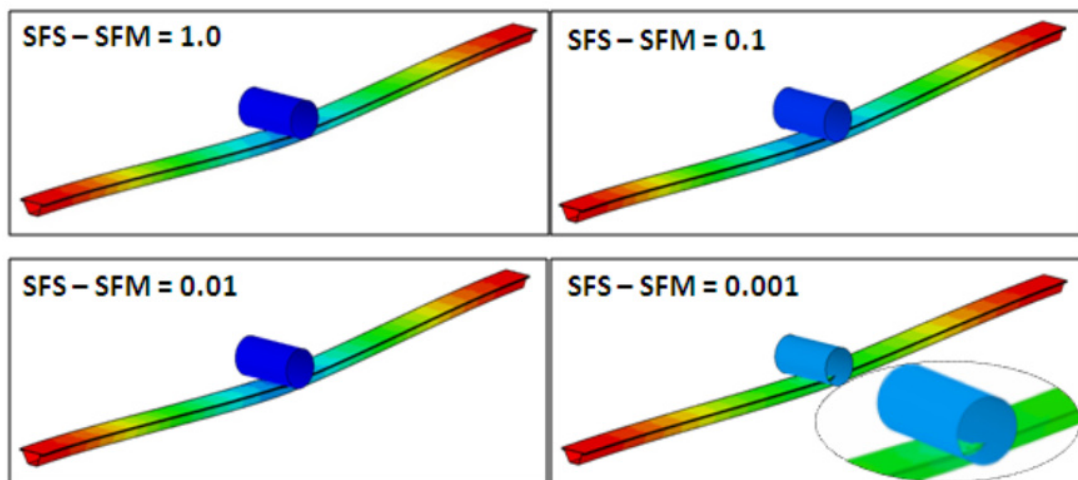

\section{$S F S-S F M=0.001$}
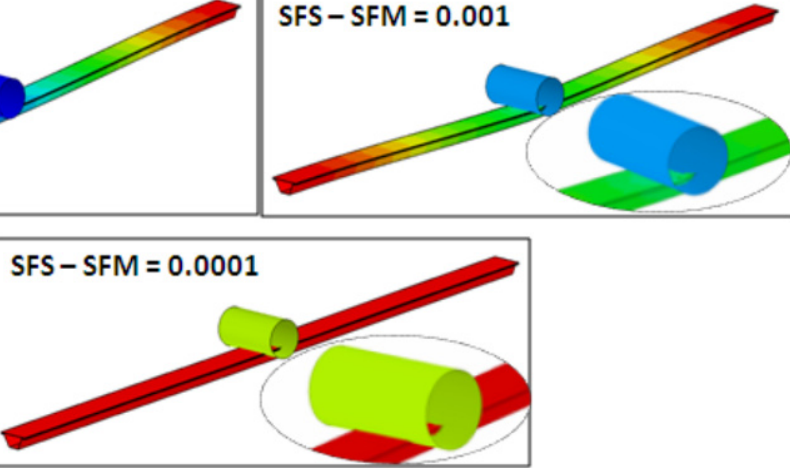

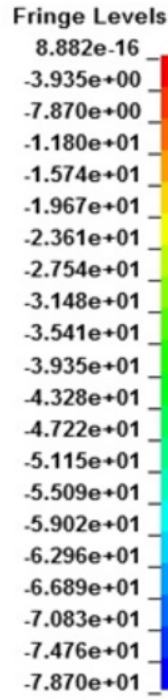

Figure 12. Effect of SFS/SFM values on contact of impactor and stiffened plate.

different SOFT parameter values. In case of SOFT $=2$, final absorbed energy is slightly higher than the other SOFT cases. The reaction force also did not change with different SOFT values (Figure 10) but by increasing the SOFT value the response becomes more oscillatory. As the SOFT $=2$ gives a result closer to the experiment, in the further analysis SOFT $=2$ is used for contact between dissimilar materials and with different mesh density. The SOFT parameter for tiebreak contact between similar mate- rials and mesh density is set according to the recommended value in LS-DYNA User Manual, i.e. SOFT $=0$.

Next the effect of the contact penalty scale factor SFS/SFM is investigated. The absorbed energy and the resultant reaction force for different SFS/SFM values is compared in Figure 11. Values of SFS in the range of 0.01 to 0.1 give acceptable results. This is in accordance with a separate study of modelling of an impacted flat plate compared with the experiments [5]. Reducing the 


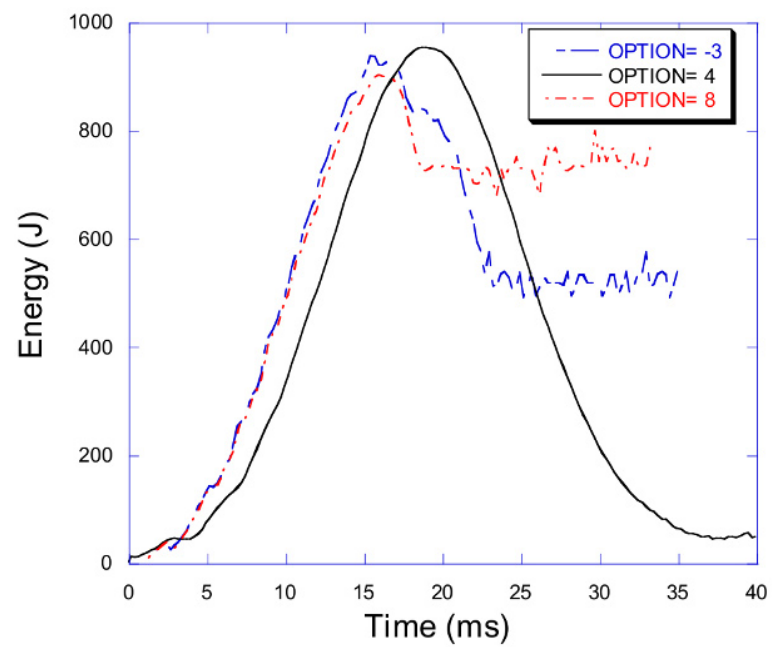

(a)

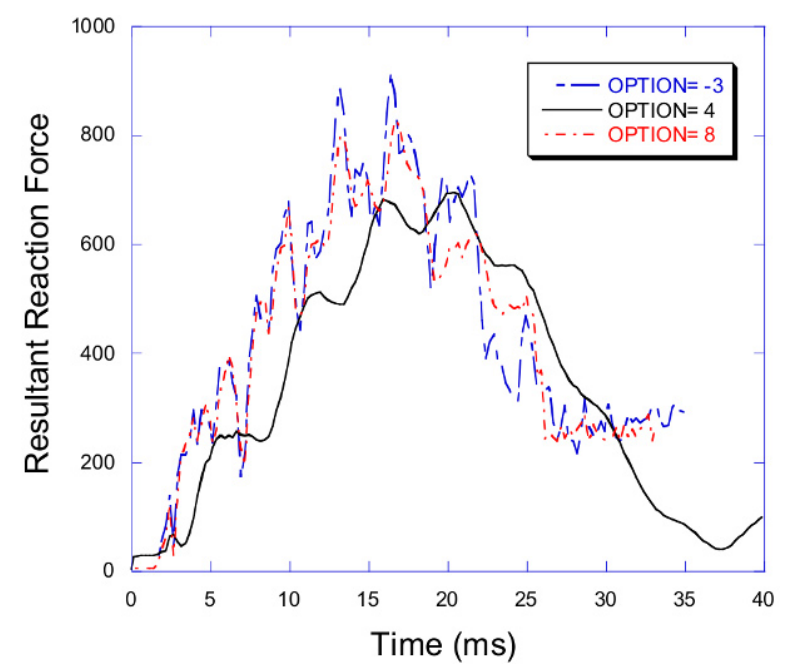

(b)

Figure 13. Comparison of (a) absorbed energy and (b) contact force of composite plate using different number of sublaminates with $\mathrm{SFS}=\mathrm{SFM}=$ 0.1 .

SFS/SFM value below 0.01 causes a forward shift to the energy curve in time, while the final absorbed energy is also reduced. This is because lower stiffness for materials means there is no quality contact between them. The impactor crashed into the plate and continued its movement in the same direction without damage to any elements of the plate. Figure 12 shows $Y$ displacement contour plot at a fixed fringe levels. Each frame is taken at the time when the displacement at the centre of the stiffened plate is at the maximum.

Behaviour of the tiebreak contact also has a significant effect on impact analysis. Contact behaviour of tiebreak contacts are defined under the OPTION parameter in $4^{\text {th }}$ mandatory card. A negative value will transfer forces and moments while positive values do not transfer moments. The use of OPTION is limited with the element type in FE model. Some of the OPTION values such as OPTION = 6 are used with only solids or thick shell. Behaviour of tiebreak contacts after failure (if a failure material model exists in FE model) is another important reason why contact parameter should be chosen carefully in addition to the type of the tiebreak contact. In this part of the paper, the OPTION parameter will be selected as $-3,4$ and 8 . When OPTION $=-3$, tiebreak contact has a general behaviour which has normal and shear components for the failure criterion as given Equation (12) and can be used with thin shell element. OPTION $=4$ can also be used with thin shell but does not contain a shear component. OPTION = 8 is the most common option for the use of tiebreak contacts for delamination analysis. _ONE_WAY_SURFACE_TO_SURFACE_TIEBREAK is the recommended contact type for this option. Different OPTION values were used in Stiffened Plate model. Comparison of the internal energy and resultant reaction force is shown in Figure 13.

In summary using tiebreak contact between sublaminates is an effective method to simulate delamination in laminated composite structures. But we must be careful in choosing the number of sublaminates, the element types in the FE model and contact types and their parameters. The energy dissipation will increase unrealistically and the results diverge from the reality in case of improper selection of the above parameters.

\section{Conclusion}

In this paper different models in the explicit finite element software LS-DYNA were developed to simulate the low velocity impact behaviour of composite plates and substructures with damageable elements and ply delamination capabilities as in modelling of laminated composites, delamination is inevitable in impact loading.

Three approaches were used for this purpose: Thick shell and solid elements with cohesive interface MAT185 interlayer and thin shell with delamination tiebreak contact. The explicit solver worked properly for the delamination tiebreak contact and with the cohesive zone interlayer in TSHELL and solid models. The results showed a good correlation with the experimental results in terms of force and energy plots.

The results of various models are compared in Figure 14. $\mathrm{FE}$ models with delamination led to satisfactory results. 


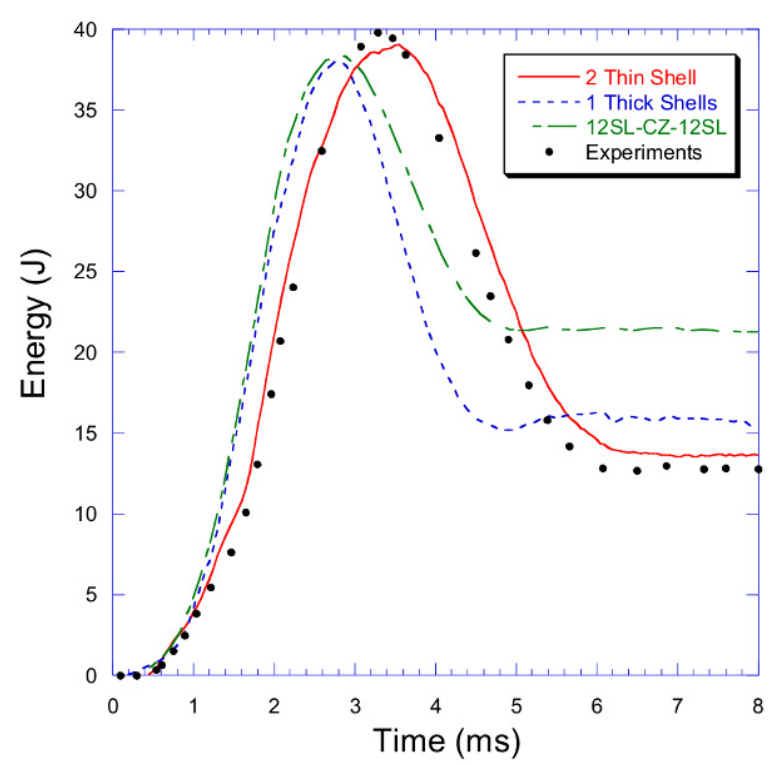

(a)

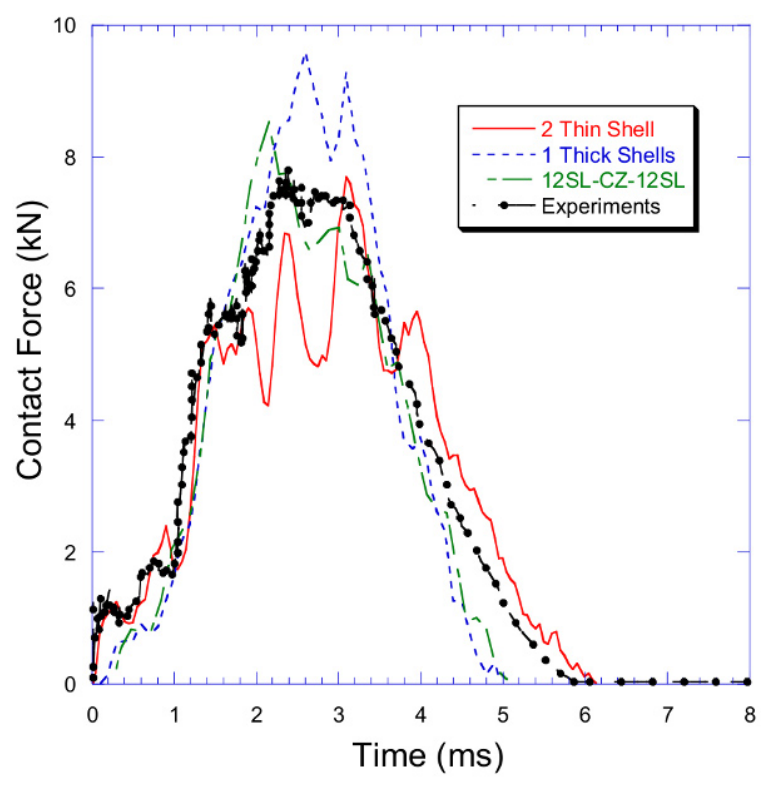

(b)

Figure 14. Comparison of internal energy and contact force of impacted plate from different modelling approaches. Experiment from [5].

Energy absorption and contact force of the plates that consists of solid elements and cohesive zone interlayers are higher than in thin shell and thick shell models.

Thin shell elements show more realistic results. The influences of contact type and contact parameters SFS/SFM, SOFT and OPTION were investigated. However the numerical simulation proved results strongly influenced by simulation parameters, in particular the element size, the number of shell sublaminates and the contact parameters.

\section{Acknowledgments}

The authors acknowledge Faculty of Science, Engineering and Computing (SEC) of Kingston University London for the financial support provided to the first author through Teaching Graduate Assistantship (TGA) scheme.

\section{References}

[1] Hadavinia H., Dogan F, Elmarakbi A., Khalili M., Modelling of low velocity impact of laminated composite substructures. International Journal of Vehicle Structures and Systems, 2011, 3(2), 96-106

[2] Lau S.T.W., Said M.R., Yaako M.Y., On the effect of geometrical designs and failure modes in composite axial crushing: A literature review, Composite Structures, 2012, 94, 803-812

[3] England J., Hadavinia H., Marchant D.R., Aboutorabi A., Design of Automotive Metal and Composite Chassis Structures, Recent Patents on Mechanical Engineering, Bentham Science, 2010, 3(3), pp. 211-225

[4] Dehkordi M.T., Nosraty H., Shokrieh M.M., Minak G., Ghelli D., Low velocity impact properties of intra-ply hybrid composites based on basalt and nylon woven fabrics, Materials \& Design. 2010; 31(8), 3835-3844.

[5] Heimbs S., Heller S., Middendorf P., Hähnel F., Weiße J., Low velocity impact on CFRP plates with compressive preload: Test and modelling., International Journal of Impact Engineering, 2009, 36(10-11), 11821193

[6] Chang F.-K., Chang K.-Y., A progressive damage model for laminated composites containing stress concentrations. J. Compos. Mater. 1987, 21, 834-855

[7] Choi H.Y., Wu H.-Y.T.. Chang F.K., A new approach toward understanding damage mechanisms and mechanics of laminated composites due to low-velocity impact: Part II-Analysis, J. Compos. Mater. 1991, 25, 1012-1038

[8] Choi H.-Y., Wang H.S., Chang F.-K., Effect of laminate configuration and impactor's mass on the initial impact damage of graphite/epoxy composite plates due to line loading impact, J. Compos. Mater. 1992, 26(6), 804-827 
[9] LS-DYNA Theory Manual, Livermore Software Technology Corporation, California, USA, LS-DYNA 971 R6; 2006

[10] Hellen T.K., On the method of the virtual crack extension, Int. J. Numer. Meth. Eng., 1975, 9:187-207

[11] Rice J.R., A path independent integral and the approximate analysis of strain concentration by notches and cracks, J. Appl. Mech., 1968, 35:379-386

[12] Rybicki E.F., Kanninen M.F., A finite element calculation of stress intensity factors by a modified crack closure integral, Eng. Fract. Mech., 1977, 9:931-938

[13] Raju I.S., Calculation of strain-energy release rates with higher order and singular finite elements, Eng. Fract. Mech., 1987, 28(3), 251-274

[14] Parks D.M., A stiffness derivative finite element technique for determination of crack tip stress intensity factors, Int. J. Fract., 1974, 10(4), 487-502

[15] Griffith A.A., The phenomena of rupture and flow in solids. Philosophical Transactions of the Royal Society, London; Series A, 1921, 221, 163-198

[16] Gordnian K., Hadavinia H., Mason P.J., Madenci E., Determination of fracture energy and cohesive strength in Mode I delamination of angle-ply laminated composites, Compos. Struct., Vol. 82(4), 577-586, 2008

[17] Hillerborg A., Modeer M., Peterson P.E., Analysis of crack formation and growth in concrete by means of fracture mechanics and finite elements, Cement Concr. Res., 1976, 6, 773-782

[18] Needleman A., A continuum model for void nucleation by inclusion debonding, J. Appl. Mech., 1987, 54, 525-531

[19] Tvergaard V., Hutchinson J.W., The relation between crack growth resistance and fracture process parameters in elastic-plastic solids, J. Mech. Phys. Solid, 1992, 40, 1377-1397

[20] Tvergaard V., Hutchinson J.W., The influence of plasticity on mixed mode interface toughness, J. Mech. Phys. Solid, 1993, 41, 1119-1135

[21] Hutchinson J.W., Linking scale in fracture mechanics. In: Proceedings of the $9^{\text {th }}$ International Conference on Fracture (ICF9), Sydney, 1-5 April 1997, 1-14

[22] Camacho G.T., Ortiz M., Computational modeling of impact damage in brittle materials, Int. J. Solid. Struct., 1996, 33, 2899-2938

[23] Mi Y., Crisfield M.A., Davies G.A.O., Hellweg H.B., Progressive delamination using interface elements, J. Compos. Mater., 1998, 32(14), 1246-1272

[24] Hillerborg A., Application of fictitious crack model to different types of materials, Int. J. Fract., 1991, 51, 95-102

[25] Dugdale D.S., Yielding of steel sheets containing slits, J. Appl. Mech., 1960, 8, 100-104

[26] Williams J.G., Hadavinia H., Analytical solution of cohesive zone models, J. Mech. Phys. Solid., 2002, 809-825

[27] Chen J., Crisfield M., Kinloch A.J., Busso E.P., et al., Predicting progressive delamination of composite materials specimens via interface elements, Mech. Compos. Mater. Struct., 1999, 6,1-17

[28] Blackman B.R.K., Hadavinia H., Kinloch A.J., Williams J.G., The use of cohesive zone model to study the fracture of fibre composites and adhesively-bonded joints, Int. J. Fract., 2003, 119(1), 25-46

[29] Elmarakbi A.M., Hu N., Fukunaga H., Finite element simulation of delamination growth in composite materials using LS-DYNA, Compos. Sci. Tech., 2009, 69(14), 2383-2391

[30] Barenblatt G.I., The formation of equilibrium cracks during brittle fracture. General ideas and hypotheses: axially-symmetric cracks, J. Appl. Math. Mech. (PMM), 23, 434-444, 1959

[31] Rice J.R., Wang J-S., Embrittlement of interfaces by solute segregation, Mat. Sci. Eng. A, 1989, 107, 23-40

[32] Xu X.P., Needleman A., Numerical simulation of fast crack growth in brittle solids, J. Mech. Phys. Solid., 1994, 42, 1397-1434

[33] Geubelle P.H., Baylor J., Impact-induced delamination of laminated composites: a 2D simulation, Compos. B Eng., 1998, 29, 589-602

[34] Pandya K., Williams J.G., Measurement of cohesive zone parameters in tough polyethylene, Polymer Engineering and Science, 2000, 40(8), 1765-1776

[35] Mohammed I., Leichti K.M., Cohesive zone modelling of crack nucleation at bimaterial corners, J. Mech. Phys. Solid., 2000, 48 ,735-764

[36] Hughes T.J.R., Taylor R.L., Sackman J.L., Curnier A.C., et al., A Finite Element Method for a Class of ContactImpact Problems, 1976

[37] Belytschko T., Yeh I. S., The splitting pinball method for contact-impact problems, Comput. Meth. Appl. Mech. Eng., 1993, 105(3), 375-393

[38] Burton D.E., Physics and Numerics of the TENSOR Code, Lawrence Livermore National Laboratory, Internal Document, UCID-19428, 1982

[39] Wilkins M. L., Calculations of Elastic-Plastic Flow, 1964 